\title{
Screening of Epigallocatechin-3-Gallate (EGCG) Content in Camellia sinensis Products
}

Nurul Ain Syukriyah Ahmad Muhamud and Syazwaniitri Amran*

Department of Biosciences, Faculty of Biosciences and Medical Engineering, Universiti Teknologi Malaysia, Malaysia

*Corresponding author: Syazwaniitri Amran, Professor, Department of Biosciences, Faculty of Biosciences and Medical Engineering, Universiti Teknologi Malaysia, Malaysia, Tel: +6075557762; E-mail: syazwaniitri@utm.my

Received: August 14, 2018; Accepted: August 21, 2018; Published: August 28, 2018

Copyright: ( 2018 Ahmad Muhamud NAS, et al. This is an open-access article distributed under the terms of the Creative Commons Attribution License, which permits unrestricted use, distribution, and reproduction in any medium, provided the original author and source are credited.

\begin{abstract}
Epidemiological studies have proven the influences of tea consumption in prevention of chronic diseases. Tea (Camellia sinensis) is a major source of epigallocatechin-3-gallate (EGCG) compound with pharmacological properties such as anti-obesity, anti-diabetes, anti-inflammation. The aims of this study is to analyze and compare the EGCG content in commercialized tea-based products, infusion tea and ready-to-drink tea beverages available in the local market. This study is divided into two phases. The first phase involved the extraction of EGCG compound from four types of tea namely as white tea (WT), green tea (GT), oolong tea (OT) and black tea (BT) using aqueous and methanol extraction techniques. The amount of EGCG is determined using HPLC. The second phase involved the characterization of EGCG compound in infusion tea bags (ITB) and ready-to-drink tea beverages (RTD) using HPLC. Our findings recorded that GT contain the highest EGCG concentration in comparison to other tea types using both methanol and aqueous extraction methods. Temperature and extraction time have influence on the extraction yield. This study also revealed that ready-to-drink tea beverages contains significantly lower level of EGCG compared to the infusion tea hence makes ready-to-drink beverages less healthy for consumption especially for obese and diabetics individuals.
\end{abstract}

Keywords: Tea; EGCG; TLC; HPLC; Pharmacological properties; Infusion tea

\section{Introduction}

Tea is one of the most preferable beverages among people throughout the world due to its beneficial effect towards human health. There are four major types of tea has been derived from Camellia sinensis leaves known as White tea (WT), Green tea (GT), Oolong tea (OT) and Black tea (BT) which differ in processing step. The compound responsible to provide positive effects of tea is known as EGCG, a tea catechin [1,2]. Different types of tea have different level of tea catechin [3]. The pharmacological properties of EGCG such as antioxidant, anti-obesity, anti-diabetes and anti-inflammatory makes tea as potential alternative for disease control, weight management and regulating cholesterol level [4-6].

In the market, there have been an increased number of health supplements and food products desired from tea claims to help in reducing body weight, lowering the blood cholesterol level and blood glucose level. However, the level of EGCG content may differ in different types of tea products hence, the pharmacological effect might be different. Therefore, this study presents a method to characterize the presence of EGCG in several commercialize tea-based product in order to validate its effectiveness as food supplement. This study also reveal the optimize condition to extract EGCG compound from different types of tea samples. Results from this study provide insights in empowering consumer rights and ensure the food security.

\section{Materials and Methods}

\section{Materials and reagents}

Fourteen infusion tea bag samples and twelve ready-to-drink samples were purchased randomly from the market. ECGC standard were purchased from Calbiochem with HPLC purity not less than 99.5\%. HPLC grade of Acetonitrile, methanol, acetic acid were used during extraction and HPLC analysis.

\section{Extraction procedure}

$5 \mathrm{~g}$ of the tea sample was brewed in $250 \mathrm{ml}$ methanol and $250 \mathrm{ml}$ distilled water at temperature $37^{\circ} \mathrm{C}$ for 60 minutes with continuous stirring. The tea solution was filtered using Whatman No.1 filter paper twice. Next, the extract solution was centrifuged at $4500 \mathrm{rpm}$ for 3 minutes. The supernatant was collected into conical flask and the residue was discarded. After that, the solution was dried using freeze dryer until powder is obtained. The dried extract was stored in $-20^{\circ} \mathrm{C}$ refrigerator. This method was repeated for GT and BT samples. The process was repeated twice.

\section{Optimization of aqueous extraction condition}

For optimization of temperature, $5 \mathrm{~g}$ of tea sample was brewed in $250 \mathrm{ml}$ distilled water at different temperature $\left(29^{\circ} \mathrm{C}, 50^{\circ} \mathrm{C}, 60^{\circ} \mathrm{C}\right.$ and $80^{\circ} \mathrm{C}$ ) for 60 minutes with continuous stirring. The procedure was similar as mention in Extraction procedure. The optimum temperature determined from each types of tea is used as temperature for optimization of extraction time, $5 \mathrm{~g}$ of tea sample was brewed in 250 $\mathrm{ml}$ distilled water at different extraction time $(20 \mathrm{~min}, 30 \mathrm{~min}, 40 \mathrm{~min}$ and $60 \mathrm{~min}$ ). The temperature was set based on the optimum 
temperature of the desired tea samples. The extraction procedure was conducted as mention in previous section. This method was repeated twice for WT, GT, OT and BT samples.

\section{HPLC analysis}

Qualitative and quantitative determination of EGCG was performed using High Performance Liquid Chromatography (HPLC). The chromatographic separations were carried out using Agilent C-18 column $(4.6 \times 150 \mathrm{~mm}, 5$ micron). The combination of $20 \%$ buffer A (water with $0.05 \%$ Trifluroacetic Acid (TFA)) and $80 \%$ buffer B (acetonitrile with $0.05 \%$ TFA) were used as the mobile phase and filtered using $0.20 \mu \mathrm{m}$ nylon filter. The flow rate was set at $1.0 \mathrm{ml} / \mathrm{min}$ with oven temperature maintained at $25^{\circ} \mathrm{C}$. Detection with diode array detector was used at wavelength $280 \mathrm{~nm}$. EGCG standard $(99.5 \%$ purity) was prepared in serial dilutions to generate a standard curve. The samples were prepared by diluting $5 \mathrm{mg}$ of freeze-dried extract into $1 \mathrm{ml}$ of buffer A and filtered with $0.20 \mu \mathrm{m}$ nylon filter. For infusion tea and ready-to-drink tea, $1 \mathrm{ml}$ of undiluted tea solution from each samples was tested. The presence of EGCG in tea extracts and tea samples were analyzed by comparing the chromatogram of the samples to the chromatograms of the EGCG standard while EGCG concentration from each sample was calculated using EGCG standard curve. The results were presented in mean with standard deviation.

\section{Results and Discussion}

\section{Extraction of EGCG from GT and BT using methanol extraction and aqueous extraction method}

Methanol extraction yield a higher EGCG concentration compared to aqueous extraction in both GT and BT samples. The similar result was obtained in previous study by Vuong et al. [7], extraction with methanol provide a better efficiency for catechin extraction compared to extraction with hot water only (aqueous extraction). Since EGCG is water soluble compound, it has higher solubility in water compares to organic solvent such as methanol, but it has higher polarity towards methanol which influenced it to be soluble in methanol (Table 1) [8].

\begin{tabular}{|l|l|l|l|}
\hline Extraction method & Tea type & Extraction yield (\%) & EGCG Concentration (mg/ml) \\
\hline Methanol Extraction & GT & 8.21 & $0.9347 \pm 0.08$ \\
& BT & 1.84 & $0.5385 \pm 0.12$ \\
\hline Aqueous Extraction & GT & 26.87 & $0.6705 \pm 0.09$ \\
& BT & 20.46 & $0.4410 \pm 0.12$ \\
\hline
\end{tabular}

Table 1: Comparison of EGCG extraction process using methanol extraction and aqueous extraction method.

Methanol extraction (ME) yield higher EGCG concentration compare to aqueous extraction (AQ) at the same extraction condition, $37^{\circ} \mathrm{C}$ and 60 minutes extraction continuous with continuous stirring. The result was presented with mean, $\mathrm{n}=2$ and standard deviation.

The presence of EGCG in tea samples were analyzed by comparing the retention time of tea samples to EGCG standard through HPLC results. The EGCG standard showed one sharp and high peak at retention time 2.762. The tea samples showed 2 single peaks at retention time 2.740 and 3.091. Based on the EGCG standard reference, the peak formed at retention time 2.740 minutes was indicated as EGCG while peak second peak obtained at 3.091 minutes was referred as caffeine according to Vasisht et al. [9].

EGCG standard curve is plotted with $\mathrm{R}^{2}>0.9882$ to quantify the amount of EGCG is the tea samples. High level of EGCG concentration is recorded in GT samples $(1.0958 \pm 0.14)$ followed by WT $(0.9604 \pm 0.00)$, OT $(0.7747 \pm 0.05)$ and BT $(0.7747 \pm 0.05)$. This result is in accordance with Chen et al. [1], Yi et al. [10], Demir et al. [11] which reported EGCG is abundantly found in GT.

\section{Optimization of temperature and extraction time in extraction of EGCG from WT, GT, OT and BT using aqueous extraction method}

Higher EGCG concentration is obtained using methanol extraction, however, in this study, aqueous extraction method is chosen to be optimized since water is the solvent usually used to prepare a cup of tea for daily consumption.

Extraction of EGCG by aqueous extraction is influenced by the temperature and extraction time $[7,8,12]$. From the experiment, the extraction of EGCG from WT, GT and OT are influenced by the temperature. As illustrated in Figure 1, there are a significant different between the EGCG concentration for WT, GT and OT at different temperature and showed that $80^{\circ} \mathrm{C}$ yield the highest EGCG concentration for WT $(0.9301 \pm 0.04)$, GT $(0.8835 \pm 0.09)$ and OT $(0.6949 \pm 0.04)$. However, the highest EGCG concentration for BT samples is obtained at lower temperature with EGCG concentration value of $(0.4949 \pm 0.02)$. This finding mimic previous reports by Vasisht et al. [9], Pastoriza et al. [13] and Vuong et al. [14] which $80^{\circ} \mathrm{C}$ is the best temperature to yield higher concentration of EGCG content in tea sample especially for green tea and black tea is not affected by temperature [8].

WT and GT samples were affected by the extraction time. Based on Figure 1, the concentration of EGCG increased with time for WT but inversely proportional for GT. This finding mimic previous reports where the yield of EGCG from tea increases as the extraction time increases [8]. Meanwhile, the results for GT is in synchrony with the results obtained by Pastoriza et al. [15] which indicates that the yield of EGCG from GT decrease with time. However, the amount of EGCG obtained from OT and BT are almost similar with highest EGCG concentration was extracted at 40 minutes. 


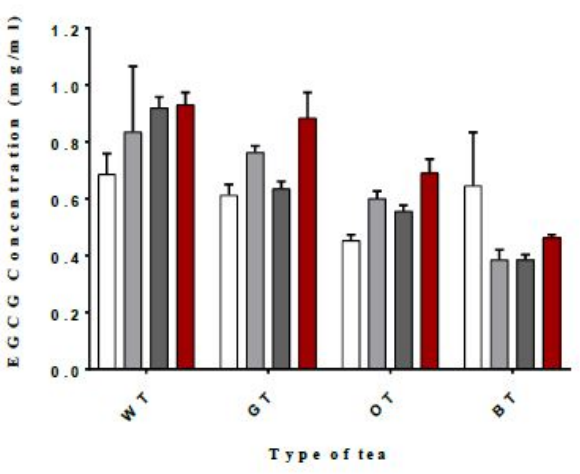

(a)

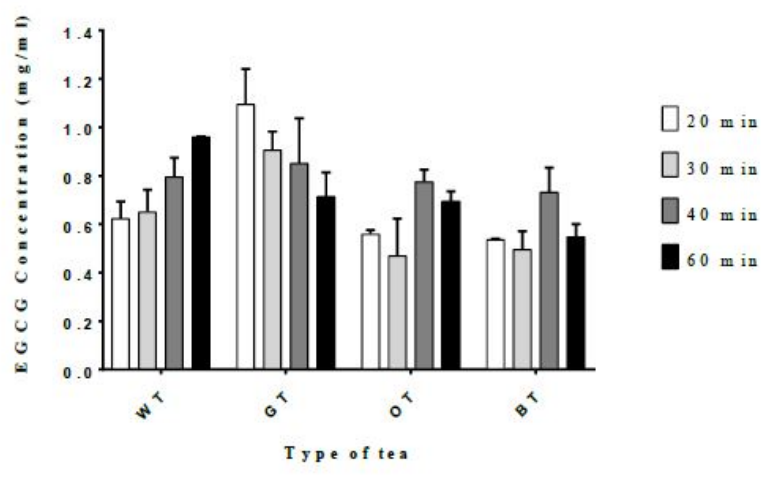

(b)

Figure 1: Optimization of aqueous extraction condition for extraction of EGCG from WT, GT, OT and BT. (a) Temperature optimization, (b) extraction time optimization. The result is presented with mean $(n=3)$ and standard deviation.

When comparing the level of EGCG in infusion tea bag (ITB) and ready-to-drink tea beverages (RTD), the EGCG concentration in ITB is much higher compared to RTD. EGCG and other catechin counterparts are easily disrupted by heat, light and chemicals which makes EGCG content in RTD beverages is lesser along the manufacturing process rather than ITB which undergo simple fermentation process. The preparation of infusion tea suggested by manufacturer is brewing the tea in boiling water for 3 minutes may facilitated the extraction of more catechin. The result is in agreement with Oliveira [15] where by EGCG content in ITB is higher compare to RTD beverages.
Besides, different brands of tea products contain different level of EGCG content which is depends on the processing methods hence the price reflect the quality of the tea brand in the market. Based on Table 2, there are a significant different between the level of EGCG in different brands of WT, GT, OT and BT products which reflects its value for health. Same results were obtained in Table 3 where the difference among the brands is significant in GT and BT products.

\begin{tabular}{|c|c|c|c|c|}
\hline Types of tea & Brands & Number of sachets/mg & $\begin{array}{l}\text { Price/number of } \\
\text { sachets (RM) }\end{array}$ & EGCG concentration $(\mathrm{mg} / \mathrm{ml})$ \\
\hline \multirow[t]{2}{*}{ WT } & A & $1 / 2 \mathrm{mg}$ & $29.90 / 25$ & $0.2246 \pm 0.034$ \\
\hline & B & $1 / 1.8 \mathrm{mg}$ & $24.50 / 20$ & $0.4715 \pm 0.057$ \\
\hline \multirow[t]{4}{*}{ GT } & C & $1 / 2 \mathrm{mg}$ & $5.40 / 25$ & $0.7222 \pm 0.082$ \\
\hline & $\mathrm{D}$ & $1 / 2 \mathrm{mg}$ & $11.60 / 25$ & $0.8741 \pm 0.249$ \\
\hline & $\mathrm{E}$ & $1 / 2 \mathrm{mg}$ & $8.90 / 25$ & $0.4619 \pm 0.252$ \\
\hline & $\mathrm{F}$ & $1 / 2 \mathrm{mg}$ & $9.75 / 25$ & $0.7106 \pm 0.076$ \\
\hline \multirow[t]{3}{*}{ от } & G & $1 / 5 \mathrm{mg}$ & $24.00 / 52$ & $0.3516 \pm 0.057$ \\
\hline & $\mathrm{H}$ & $1 / 2 \mathrm{mg}$ & $15.30 / 25$ & $0.3737 \pm 0.020$ \\
\hline & $\mathrm{F}$ & $1 / 2 \mathrm{mg}$ & $9.75 / 25$ & $0.7496 \pm 0.004$ \\
\hline \multirow[t]{4}{*}{ BT } & I & $1 / 2 \mathrm{mg}$ & $3.10 / 20$ & $0.2626 \pm 0.097$ \\
\hline & $\mathrm{J}$ & $1 / 2 \mathrm{mg}$ & $6.50 / 25$ & $0.3750 \pm 0.087$ \\
\hline & $\mathrm{K}$ & $1 / 2 \mathrm{mg}$ & $2.30 / 20$ & $0.2668 \pm 0.034$ \\
\hline & $\mathrm{D}$ & $1 / 2 \mathrm{mg}$ & $2.70 / 25$ & $0.2845 \pm 0.025$ \\
\hline
\end{tabular}




\section{$\mathrm{E}$} $1 / 2 \mathrm{mg}$ 10.55

$0.3719 \pm 0.049$

Table 2: EGCG concentration in ready-to-drink tea beverages. The result is presented with mean $(\mathrm{n}=3)$ and standard deviation.

\begin{tabular}{|c|c|c|c|}
\hline Types of tea & Brands & Price per 250 ml (RM) & EGCG concentration $(\mathrm{mg} / \mathrm{ml})$ \\
\hline WT & $\mathrm{N}$ & 2.50 & $0.0762 \pm 0.007$ \\
\hline \multirow[t]{5}{*}{ GT } & $\mathrm{L}$ & 2.50 & $0.1534 \pm 0.005$ \\
\hline & $M$ & 1.80 & $0.3196 \pm 0.010$ \\
\hline & $\mathrm{N}$ & 2.50 & $0.2967 \pm 0.004$ \\
\hline & $\mathrm{O}$ & 1.90 & $0.1825 \pm 0.002$ \\
\hline & $\mathrm{P}$ & 2.20 & $0.1564 \pm 0.040$ \\
\hline OT & $\mathrm{N}$ & 2.50 & $0.1182 \pm 0.008$ \\
\hline \multirow[t]{5}{*}{ BT } & $\mathrm{L}$ & 2.50 & $0.0503 \pm 0.004$ \\
\hline & M & 1.80 & $0.2626 \pm 0.008$ \\
\hline & $\mathrm{N}$ & 2.50 & $0.1982 \pm 0.031$ \\
\hline & $\mathrm{O}$ & 1.90 & $0.1048 \pm 0.001$ \\
\hline & $\mathrm{P}$ & 2.20 & $0.1358 \pm 0.006$ \\
\hline
\end{tabular}

Table 3: EGCG concentration in ready-to-drink tea beverages $(n=3)$.

\section{Conclusion}

In conclusion, the optimum extraction condition of white tea, green tea, oolong tea and black tea is different from each other. The different was influenced by the fermentation step during tea processing. GT contain higher EGCG content compare to the other types of tea, WT, OT and BT. The extraction of EGCG compound from WT, GT and OT were influenced by temperature but not for $\mathrm{BT} .80^{\circ} \mathrm{C}$ is the best temperature for to extract EGCG from WT, GT and OT using aqueous extraction. Apart from that, higher EGCG content is extracted at shorter time for GT but longer time is needed for WT. Both EGCG from OT and BT samples were best extracted at 40 minutes.

The level of EGCG content per $\mathrm{ml}$ is much lower compare to infusion tea hence, it provide less benefit for health. Besides, different brands of tea products contain different EGCG content depends on the processing methods hence the price reflect the quality of the tea brand in the market. Ready-to-drink tea beverage is an unhealthy option for drinks. Therefore, it is recommended to drink infusion tea especially GT at least three cups per day to get the benefits of EGCG towards health. However, further study and research are needed to evaluate the effectiveness of tea consumption in treatment and prevention of obesity for normal, overweight and obese people.

\section{Acknowledgements}

This work is supported by the Faculty of Biosciences and Medical Engineering, Universiti Teknologi Malaysia.

\section{References}

1. Chen D, Milacic V, Chen MS, Wan SB, Lam WH, et al. (2008) Tea polyphenols, their biological effects and potential molecular targets. Histology and Histopathology 23: 487-496.

2. Forester SC, Lambert JD (2011) Antioxidant effects of green tea. Molecular Nutrition and Food Research 55: 844-854.

3. Jain A, Manghani C, Kohli S, Nigam D, Rani V (2013) Tea and human health: the dark shadows. Toxicol Lett 220: 82-87.

4. Othman AI, El-Sawi MR, El-Missiry MA, Abukhalil MH (2017) Epigallocatechin-3-gallate protects against diabetic cardiomyopathy through modulating the cardiometabolic risk factors, oxidative stress, inflammation, cell death and fibrosis in streptozotocin-nicotinamideinduced diabetic rats. Biomed Pharmacother 94: 362-373.

5. Oyama JI, Shiraki A, Nishikido T, Maeda T, Komoda H, et al. (2017) EGCG, a green tea catechin, attenuates the progression of heart failure induced by the heart/muscle-specific deletion of MnSOD in mice. J Cardiol 69: 417-427.

6. Sampath C, Rashid MR, Sang S, Ahmedna M (2017) Green tea epigallocatechin 3-gallate alleviates hyperglycemia and reduces advanced glycation end products via nrf2 pathway in mice with high fat dietinduced obesity. Biomedicine and Pharmacotherapy 87: 73-81.

7. Vuong QV, Golding JB, Stathopoulos CE, Nguyen MH, Roach PD (2011) Optimizing conditions for the extraction of catechins from green tea using hot water. J Sep Sci 34: 3099-3106.

8. Lee KJ, Lee SH (2008) Extraction behavior of caffeine and EGCG from green and black tea. Biotechnology and Bioprocess Engineering 13: 646-649.

9. Vasisht K, Pritam Dev Sharma MK, Dutt Rakesh D, Vyas S, Sethi S, et al. (2003) Study to promote the industrial exploitation of green tea polyphenols in India. Trieste, Italy: United Nation Industrial Development Organization and the International Centre for Science and High Technology. 
Citation: Ahmad Muhamud NAS, Amran S (2018) Screening of Epigallocatechin-3-Gallate (EGCG) Content in Camellia sinensis Products. Nat Prod Chem Res 6: 339. doi:10.4172/2329-6836.1000339

Page 5 of 5

10. Yi T, Zhu L, Peng WL, He XC, Chen HL, et al. (2015) Comparison of ten major constituents in seven types of processed tea using HPLC-DAD-MS followed by principal component and hierarchical cluster analysis. LWTFood Science and Technology 62: 194-201.

11. Demir E, Serdar G, Sökmen M (2015) Comparison of Some Extraction Methods for Isolation of Catechins and Caffeine from Turkish Green Tea. International Journal of Secondary Metabolite 2: 16-25.

12. Amra PU, Škerget A, Knez M, Weinreich Ž, Otto B, et al. (2006) Extraction of active ingredients from gren tea (Camellia sinensis): Extraction efficiency of major catechins and caffeine. Food Chemistry 96: 597-605.
13. Pastoriza S, Pérez-Burillo S, Rufián-Henares JÁ (2017) How brewing parameters affect the healthy profile of tea. Current Opinion in Food Science 14: 7-12

14. Vuong Q, Golding J, Nguyen M, Roach P (2010) Extraction and isolation of catechins from tea. Journal of Separation Science 33: 3415-3428.

15. Oliveira RMMD (2012) Quantification of catechins and caffeine from green tea (Camellia sinensis) infusions, extract, and ready-to-drink beverages. Food Science and Technology (Campinas) 32: 163-166. 